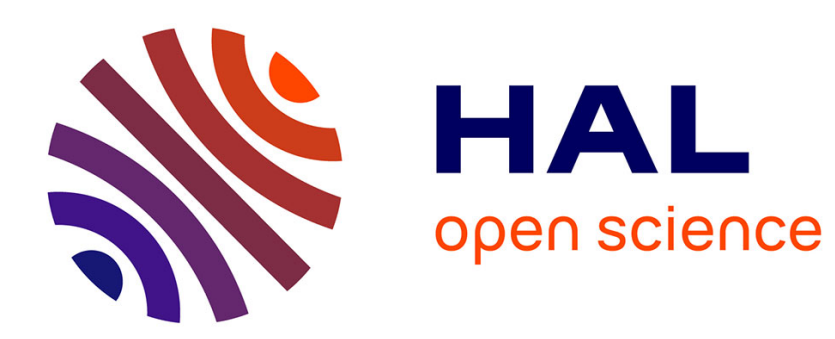

\title{
Réflexion sur les problèmes financiers de l'enrésinement
}

G. Rabouille

\section{- To cite this version:}

G. Rabouille. Réflexion sur les problèmes financiers de l'enrésinement. Revue forestière française, 1967, 2, pp.94-97. 10.4267/2042/24876 . hal-03389992

\section{HAL Id: hal-03389992 \\ https://hal.science/hal-03389992}

Submitted on 21 Oct 2021

HAL is a multi-disciplinary open access archive for the deposit and dissemination of scientific research documents, whether they are published or not. The documents may come from teaching and research institutions in France or abroad, or from public or private research centers.
L'archive ouverte pluridisciplinaire HAL, est destinée au dépôt et à la diffusion de documents scientifiques de niveau recherche, publiés ou non, émanant des établissements d'enseignement et de recherche français ou étrangers, des laboratoires publics ou privés. 


\section{RÉFLEXION \\ SUR LES PROBLEMES FINANCIERS \\ DE L'ENRÉSINEMENT}

PAR

G. RABOUILLE

D.R.O.N.F. à Châlons-sur-Marne (Marne)

L'enrésinement d'une forêt pose un certain nombre de problèmes dont il faut avoir une vue nette pour pouvoir orienter chaque cas particulier vers la solution qui lui est propre.

Trop souvent, le mot enrésinement est devenu un cliché qui recouvre des réalités que les intéressés ne distinguent plus suffisamment.

Nous croyons qu'il y a deux caractéristiques du placement forestier dans un enrésinement qu'il faut bien considérer avant de se lancer dans un travail d'envergure.

D'abord, et c'est peut-être une vérité de La Palisse, l'investissement consacré pour un enrésinement est GELÉ pendant la période s'étendant de la plantation au moment où le peuplement peut comporter une réalisation importante. Ainsi donc, le planteur doit savoir qu'il ne pourra pas retirer son argent de l'opération avant 40 ans.

Des efforts ont été tentés de manière à pouvoir mobiliser le cas échénnt le capital investi. c'est-à-dire en principe à vendre tout ou partie de la narcelle considérée, mais dans les circonstances actıelles, s'il est loisible de penser au'une personne puisse envisager d'immobiliser son capital pour une durée de 5 à 10 ans, les personnes qui se sentent assez sûres de l'avenir et qui ont un sens suffisant de la continuité des choses pour admettre un placement à un terme sinnérieur à 10 ans, ne snnt guère nombreuses. L'exnérience prouve qu'il est relativement difficile de placer des parts d'une affaire qui ne paiera de retour qu'après plus de 10 ans.

La personne a11 se fera donc engagée initialement dans une onération à immobilisation à 40 ans. aura du mal à trouver les relais nécessaires pour mobiliser, si elle en a besoin, tout ou partie de 
son investissement. Le groupement forestier dont l'activité serait exclusivement consacrée à la plantation de terrains nus, pourra sûrement trouver des souscripteurs initiaux, mais il sera difficile à ceux-ci, le cas échéant, de vendre leurs parts à un prix normal.

De tels groupements peuvent entreprendre l'enrésinement systématique d'une forêt, encore est-il que ces groupements se heurteront dans cette action à une seconde caractéristique de l'investissement d'enrésinement qui est le fait que l'investissement initial doit être ensuite réitéré pendant les années suivantes: “ IL EST DONC CUMULATIF », et son fruit ne pourra commencer à être cueilli qu'après l'échéance ci-dessus annoncée, c'est-à-dire 40 ans.

Notre attention a été attirée sur ce problème en examinant les possibilités d'un aménagement communal d'enrésinement systématique d'une forêt. La forêt était un taillis sous futaie à la révolution de 30 ans, la solution semblait être de continuer les coupes de taillis sous futaie pour enrésiner chaque coupe au passage, et ce, en totalité. Si la commune faisait un emprunt, même à $2 \%$ à 40 ans, l'annuité d'amortissement pour couvrir la dépense engagée à l'hectare était de $80 \mathrm{~F}$ à l'hectare, si donc l'opération était poursuivie pendant 30 ans, la $31^{\circ}$ année, le Maire se trouvait devant la situation suivante:

30 annuités à $80 \mathrm{~F}$ soit $2400 \mathrm{~F}$ au passif alors qu'une éclaircie à 30 ans et une éclaircie à 35 ans s'inscriraient seules à l'actif pour un chiffre de l'ordre de $800 \mathrm{~F}$, soit une balance négative de $1600 \mathrm{~F}$ sans ressource spéciale pour y faire face.

La solution s'imposait: porter la révolution du taillis sous futaie à 40 ans et limiter l'enrésinement par conséquent à $2 / 3$ des coupes chaque année au lieu d'une coupe entière.

La philosophie qu'on peut en tirer est qu'en matière d'amélioration forestière il faut mesurer ce que l'on fait à ses possibilités et ne pas vouloir tout faire en un jour. L'idée généralement répandue, que les améliorations forestières peuvent se faire du jour au lendemain, à condition d'y consacrer l'argent voulu, n'est pas raisonnable, car cet argent sera gelé pendant 40 ans et, pendant 40 ans, l'Etat, les Communes, les Particuliers, devront vivre et assurer la pérennité de leurs efforts par un financement assuré. La sagesse en pareille matière doit être de se hâter lentement en fonction de ses possibilités.

Les considérations qui précèdent éclairent les difficultés que l'on rencontre pour trouver un relais à l'investissement forestier chez les particuliers qui ne veulent plus risquer à 40 ans de distance.

Des bonifications aux capitaux ainsi investis sans rapport immédiat ont été envisagées. Nous nous rappelons avoir proposé une solution de cette sorte pour les communes de son département à 
un préfet. L'effort était raisonnable au début mais atteignait au bout des quarante premières années exactement 40 fois l'effort initial, puisqu'il était envisagé de bonifier régulièrement les intérêts des emprunts contractés pour les communes à 40 ans. Si d'ailleurs le Conseil général avait affecté une somme constante à cette bonification qui aurait pu être de $4 \%$ avec des emprunts de 40 ans, cette somme n'aurait permis que de multiplier par 25 l'effort de l'année initial puisque pendant les 39 années suivantes le crédit aurait été absorbé par la bonification consentie de $4 \%$ pendant 40 ans. Il n'a pas pu être donné suite au projet mais cet essai infructueux nous a fait toucher du doigt les difficultés de ce financement.

Il faut donc considérer que l'enrésinement est un travail de très longue haleine qu'il faut engager prudemment en ce qui concerne le particulier et que l'Etat et le marché financier, étant donné les conditions spéciales de cet investissement, sont peu aptes à soutenir pour atteindre un mouvement en rapport avec l'effort à réaliser.

Ce qui pourrait permettre de réaliser l'effort nécessaire d'enrésinement - les petits ruisseaux faisant de grandes rivières - ce seraient les efforts, jusqu'à un certain point désintéressés, des particuliers, faits pour le plaisir de voir se créer et changer une propriété pour assurer dans l'avenir, à des petits enfants, des ressources convenables à condition que les prix pratiqués régulièrement par le commerce pour les produits à en attendre, permettent d'envisager cet investissement avec un minimum de rentabilité. Or, si vers 1950 la rentabilité des plantations résineuses pour bois de mines ou bois de papeteries permettait d'envisager une rentabilité suffisante, depuis cette époque, les prix de la production de masse des plantations - bois de mines ou de papeterie - sont restés stationnaires alors que les éléments des prix de plantations ont augmenté dans des proportions considérables, notamment la main-d'œuvre. Et partout, en Allemagne, en Belgique, en France, des cris d'alarme sont poussés pour mettre en garde contre cette détérioration des prix de vente eu égard aux prix de revient.

Il est certainement difficile de mettre sur pied une organisation valable, pour lutter contre cet état de fait, l'organisation si difficile du marché du café ou du sucre en est une preuve.

Mais si l'Etat ne peut encourager de manière suffisante par le Fonds Forestier National les enrésinements indispensables à la mise en valeur du patrimoine français et si l'incitation au reboisement doit être cherché dans l'effort individuel et ce aussi bien en Allemagne et en Belgique qu'en France, il faut considérer qu'il faudra bien un jour en venir à étudier d'abord à l'échelon National puis ensuite à l'échelon Européen le problème des prix du bois de pape- 
terie et de la production du papier. L'exemple des produits agricoles de tout l'Univers montre qu'il est impossible de laisser agir en pareille matière les lois d'airain qui écrasent systématiquement les productions agricoles lorsqu'elles ne sont pas organisées.

Il est plus que temps que les Forestiers comprennent qu'ils ne vivent pas dans un monde à part mais qu'ils appartiennent au monde agricole et que les lois de ce monde agricole sont aussi bien valables pour le bois que pour le café et le sucre. 